\title{
Detection of Anaplastic Lymphoma Kinase (ALK) gene in Non-Small Cell lung Cancer (NSCLC) By CISH Technique
}

\author{
Mohanad Ali Albayyaa ${ }^{1} \&$ Ban A. Abdulmajeed ${ }^{1}$ \\ ${ }^{1}$ Department of Pathology and Forensic Medicine, Al-Nahrain University, College of Medicine, Baghdad, Iraq \\ Correspondence: Mohanad Ali Albayyaa, Department of Pathology and Forensic Medicine, Al-Nahrain \\ University, College of Medicine, Baghdad, Iraq. E-mail: Muhannedted@gmail.com
}

Received: November 21, 2017

Accepted: December 8, 2017 Online Published: January 13, 2018

doi: $10.5539 /$ cco.v7n1p22

URL: https://doi.org/10.5539/cco.v7n1p22

\begin{abstract}
Background/Aim: Anaplastic Lymphoma Kinase (ALK) rearrangement has evaluated activity of NSCLC compared with other molecular subtypes (EGFR, KRAS). Many studies demonstrated that patients with ALK rearrangement positive NSCLC have improved with a good response rates and progression (free survival) when treated with either monotherapy or by a combination therapy compared with EGFR-mutated, KRAS/EGFR/ALK wild type or KRAS-mutated. The aim of this study was to detect and study the signal pattern of normal ALK and compare it to that of mutated ALK with gene rearrangement in cases of non-small cell lung cancer and Inflammatory conditions by implementing the CISH technique. In addition to correlate ALK signal pattern with the histopathological type and grade as well as the age and sex of the patients.
\end{abstract}

Materials \& Methods: Forty patients with NSCLC and Inflammatory diseases were enrolled in a comparative cross sectional study. The tissue blocks were sectioned on non-charged slides for the preparation of routine H\&E staining. Positively charged slides were used for tissue sections prepared for chromogenic in situ hybridization procedure to detect ALK gene.

Results: ALK gene signal break apart was detected in $(18 / 20,90 \%)$ of malignant cases; $(0 / 20,0 \%)$ of non-neoplastic lung lesions. There was a significant statistical difference in their distribution, $p<0.05$. While There was no significant association between any disease status and sex P value $=1.000 \mathrm{NS}$. The CISH test was $100 \%$ sensitive using negative score as a cutoff point and $90.9 \%$ specific. The score was divided into three levels that categorized the cases, so there were six cases in score one (1-32\%), nine cases in score two (33-67\%) and three cases in score three (68-100\%).

Conclusion: Detection of ALK rearrangement in the early diagnosis of NSCLC is highly sensitive and can save a lot of efforts in planning chemotherapy regimens. Results were very promising in identifying this mutation by a sensitive and highly specific test. The ALK gene rearrangement could be an early mutation and it is needed as an initiating step for the carcinogenesis process. The presence of a double gene mutation, however, could be the cause of a higher-grade cancer.

Keywords: Anaplastic Lymphoma Kinase (ALK), Non-Small Cell Lung Cancer (NSCLC), Chromogenic In Situ Hybridization (CISH)

\section{Introduction}

Various molecular markers have been involved in the pathogenesis of lung cancer. Planning a marker study on lung cancer cases, whether this marker can differentiate a benign from malignant neoplasm and differentiate a non-small cell carcinoma (NSCLC) from small cell carcinoma, needs to consider many factors. One of these factors, is the signal score and pattern affected by the grade, stage or morphological type of the malignant neoplasm. Among the common molecular markers applied in lung cancers is Anaplastic Lymphoma Kinase (ALK) receptor tyrosine kinase which has shown to have different mutational types in the carcinogenesis of these malignancies (Christine et al., 2016; Kwak et al., 2010). Studies have evaluated activity of ALK rearrangement positive NSCLC cohorts compared with other molecular subtypes (EGFR, KRAS) (Morris et al., 2016). A small retrospective study demonstrated that patients with $A L K$ rearrangement positive NSCLC have improved with a good response rates and progression (free survival) when treated with either monotherapy or by a combination therapy compared with $E G F R$-mutated, KRAS/EGFR/ALK wild type or KRAS-mutated (Kim et al., 
2011). In contrast, a larger retrospective analysis has reported a lower response rate in $A L K$-fusion positive NSCLC and a shorter time to progression. Whether Positive $A L K$ rearrangement in NSCLC is more sensitive to pemetrexed than unselected NSCLC cohort is still a topic for investigation (Zochbauer-Muller \& Minna, 2002).

The aims of this study are to detect and study the signal pattern of normal ALK and compare it to that of mutated ALK with gene rearrangement in cases of NSCLC by implementing the CISH technique; to correlate ALK signal pattern with the histopathological type and grade of non-small cell lung cancer as well as the age and sex of the patients; and to detect the signal pattern of normal and mutated ALK that has gene rearrangement in cases of inflammatory conditions by implementing the CISH technique.

\section{Materials and Methods}

This is a comparative cross sectional study that has been conducted during the period from February 2015 to February 2016. The study has two parts.

The Study involved the collection of 40 Formalin fixed paraffin embedded lung cancer specimens and non-cancerous lung tissues as controls.

Specimens were selected after reviewing archival files of the laboratories of the specialized surgery Hospital, Oncology Hospital and Medical City directorate in addition to one of the acknowledged private laboratories.

Specimens included 27 bronchial biopsies and 13 lobectomies, Non-cancerous tissue specimens were all biopsies.

Part one: Retrospective part, whereby paraffin embedded tissue blocks of thirteen cases of lung cancer and tissue blocks of nine benign resected lung lesions were selected from the archives of the histopathology laboratory section of the Hospital of Specialized Surgeries at Baghdad Medical City. Demographic data for each case was collected from the hospital patient information records.

Part two: Prospective part, whereby biopsies were collected from seven patients having lung cancer and from 11 patients having chronic inflammatory lung diseases. After processing and examining the collected samples, histopathological data concerning the diagnosis and grades of lung cancer patients were recorded.

The tissue blocks were sectioned on non-charged slides for the preparation of routine H\&E staining. Positively charged slides were used for tissue sections prepared for chromogenic in situ hybridization procedure to detect ALK gene (ZytoDot, 2015).

\section{Kit and Probe: (ZytoDot, 2015)}

CISH working solution / Zytovision / Germany. The ZytoDot ${ }^{\circledR}$ 2C SPEC ALK Break Apart Probe is designed to detect rearrangements involving the chromosomal region 2p23 harboring the ALK (anaplastic lymphoma receptor tyrosine kinase, a.k.a. CD246) gene, the probe was purchased from Zytovision / Germany.

The ZytoDot ${ }^{\circledR}$ 2C SPEC ALK Break Apart Probe is a mixture of a Digoxigenin-labeled probe and a Dinitrophenyl labeled probe hybridizing to the $2 \mathrm{p} 23$ band.

The Digoxigenin-labeled probe hybridizes proximal to the ALK gene at 2p23, the Dinitrophenyl-labeled fluorochrome labeled probe hybridizes distal to the ALK gene at $2 \mathrm{p} 23$.

\section{Scoring and Pattern of ALK Break-Apart Dual-Color CISH:}

In each section, one hundred cells were counted. The positive ALK rearrangement was diagnosed when distance between the green and red signals exceed the size of one signal. The pattern of positive signals of ALK rearrangement included 2 types: pattern -1 , when there were 2 red and 2 green signals (separated by a distance more than a signal size). This suggested the rearrangement involving the 2 gene copies ( 2 alleles). The pattern -2 was considered when only one red and one green signals (separated by distance larger than a signal size) together with a brown signal or (very close red and green signals). This suggested one gene copy rearrangement. In addition to the mixed pattern between $1 \& 2$.

Three percentage scores for positive cases were adopted (Hans-Ulrich et al., 2013), by using 3D HISTECH Digital.

Score $-1: \quad 1-30 \%$ of cells

Score $-2: \quad 31-60 \%$ of cells

Score $-3:>60 \%$ 


\section{Results}

In this study, 20 cases of NSCLC and 20 cases of non-malignant patients were studied.

The mean age of patients in the carcinoma group was $59.2+-11.87$ and that for those in the inflammatory group was 50.1+- 12.64. There was a significant difference between the mean age of the two groups $p<0.05$.

Furthermore the patients in both groups were classified according to their age below 50 years or above 50 years.

Four patients with carcinoma $(20 \%)$ were below 50 years and $16(80 \%)$ were above 50 years. In the benign group $10(50 \%)$ were below 50 years and $10(50 \%)$ were above 50 years.

There was a significant statistical difference in their age distribution, $\mathrm{p}<0.05$.

ALK gene breakapart signal positivity was detected in $(18 / 20,90 \%)$ of malignant cases; $(0 / 20,0 \%)$ of non-neoplastic lung lesions (Inflammatory). There was a significant statistical difference in their distribution, $\mathrm{p}<$ 0.05 as shown in table-1\&2.

While There was no significant association between any disease status and sex; P value $=1.000^{\mathrm{NS}}$ as shown in figure-1.

Table 1. The mean, median and other statistical parameters of the age of patients both in malignant and inflammatory groups

\begin{tabular}{lll}
\hline Age & Inflammatory & NSCLC \\
\hline Mean & 50.1 & 59.2 \\
Median & 50 & 58 \\
Standard Error of Mean & 2.83 & 2.65 \\
Standard Deviation & 12.64 & 11.87 \\
Maximum & 71 & 81 \\
Minimum & 28 & 36 \\
\hline $\mathrm{p}<0.05$ & &
\end{tabular}

Table 2. Frequency distribution of lung cancer patients according to the age groups

\begin{tabular}{|c|c|c|c|c|c|c|}
\hline & & \multicolumn{2}{|c|}{ Inflammatory } & \multicolumn{2}{|c|}{ NSCLC } & \multirow[t]{2}{*}{ P value } \\
\hline & & Count & $\%$ & Count & $\%$ & \\
\hline \multirow[t]{2}{*}{ Age groups } & $<50$ years & 10 & $50 \%$ & 4 & $20 \%$ & \multirow{2}{*}{$0.046^{*}$} \\
\hline & $>50$ years & 10 & $50 \%$ & 16 & $80 \%$ & \\
\hline
\end{tabular}

NSCLC: Non-small cell lung cancer

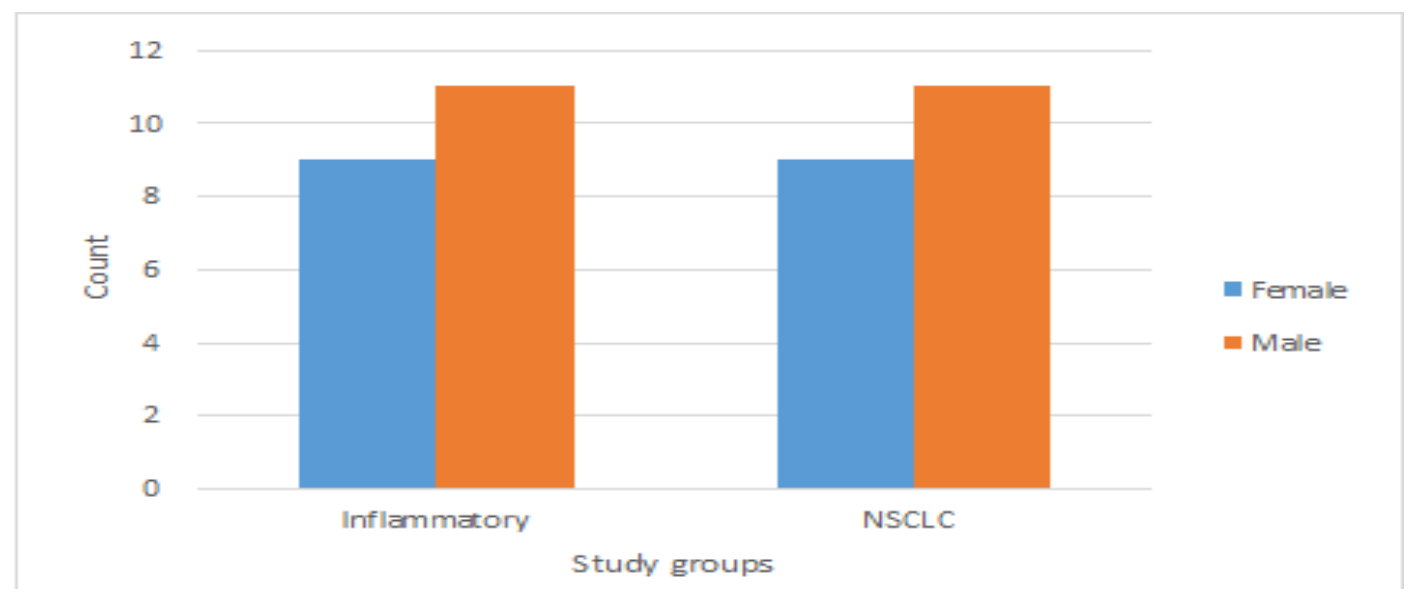

Figure 1. The sex distribution of patients in both study groups

Positive ALK CISH break apart signals were detected as either two green and two red signals separated by a distance larger than that of the signals size, or 1 green and 1 red signals separated by a signals more than the signals size together with one brown and / or red, and green signals not separated from each other according to the kit instructions as shown in figure-2 A\&B. Malignant tumors revealed positive ALK break apart signals in 18 
$(90 \%)$ cases. The other $2(10 \%)$ revealed normal signal patterns.

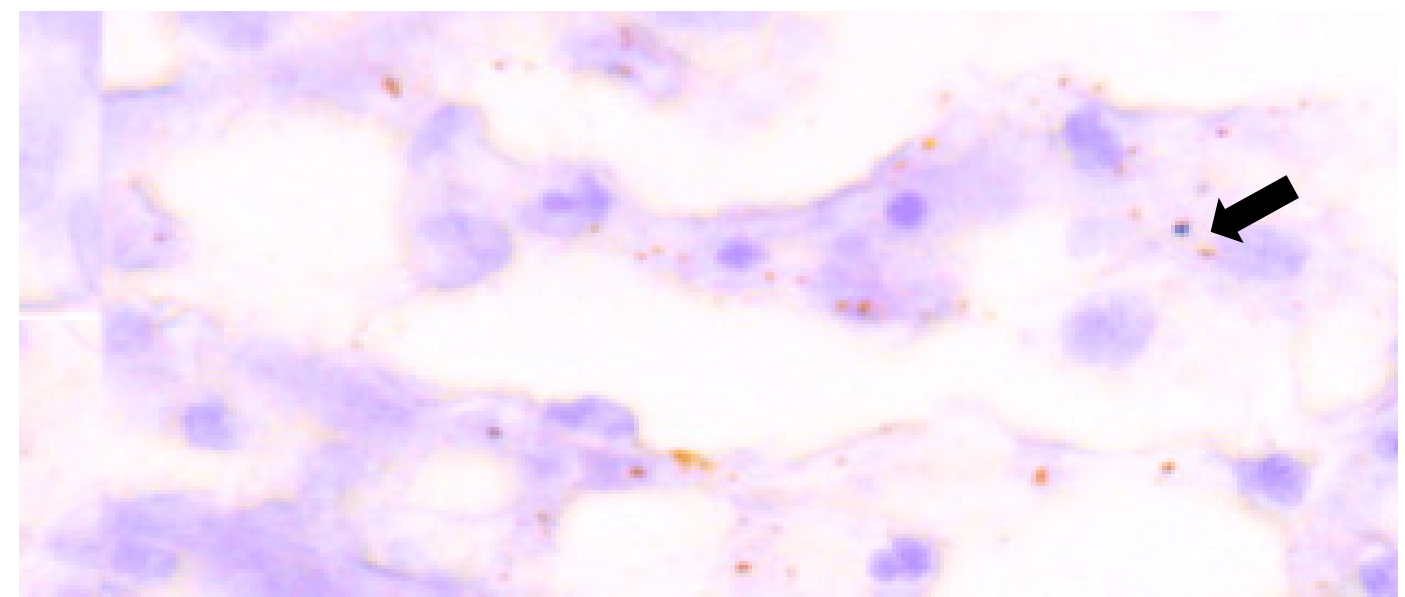

Figure 2A. Chromogenic In Situ Hybridization for ALK gene signals in Adenocarcinoma (Digital 40X). Arrow shows the break apart

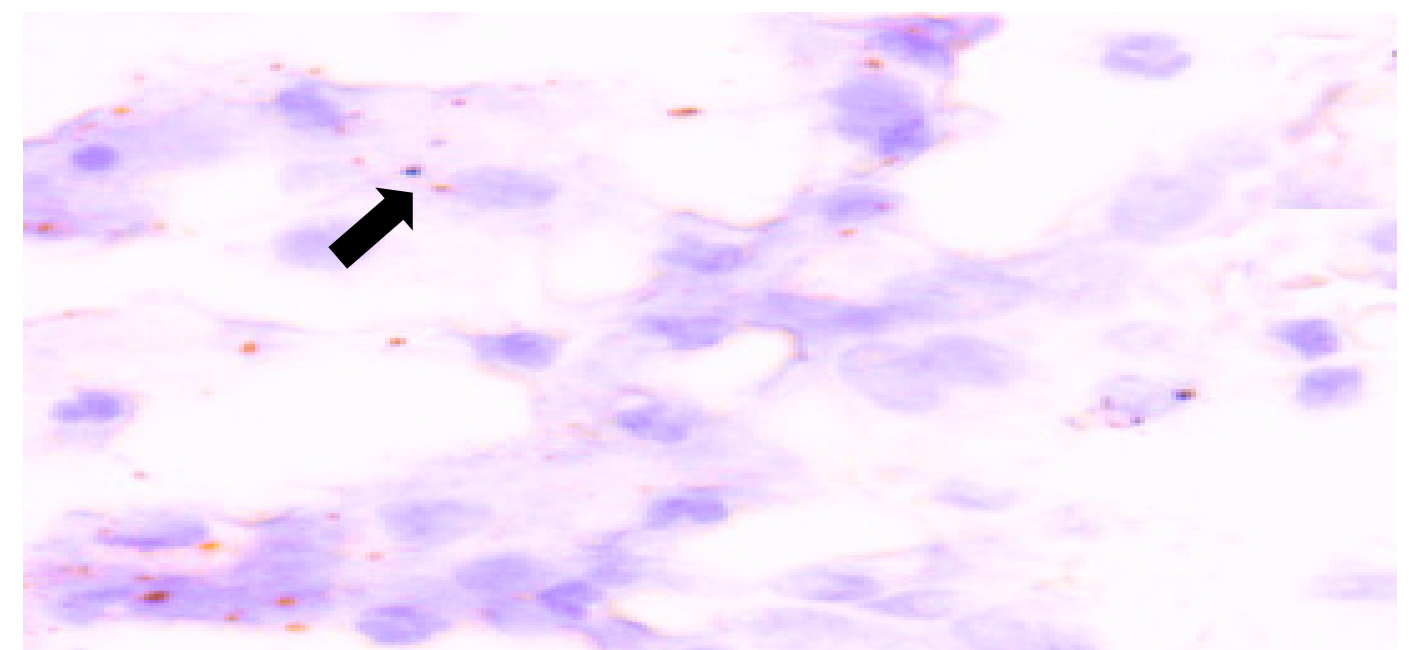

Figure 2B. Chromogenic In Situ Hybridization for ALK gene signals in Adenocarcinoma (Digital Pathology 40X), Arrow shows the break apart

Negative results for ALK break apart were detected either as non-separated red + green signals or brown signal, Benign lesions revealed normal signals patterns in all cases as shown in figure- 3

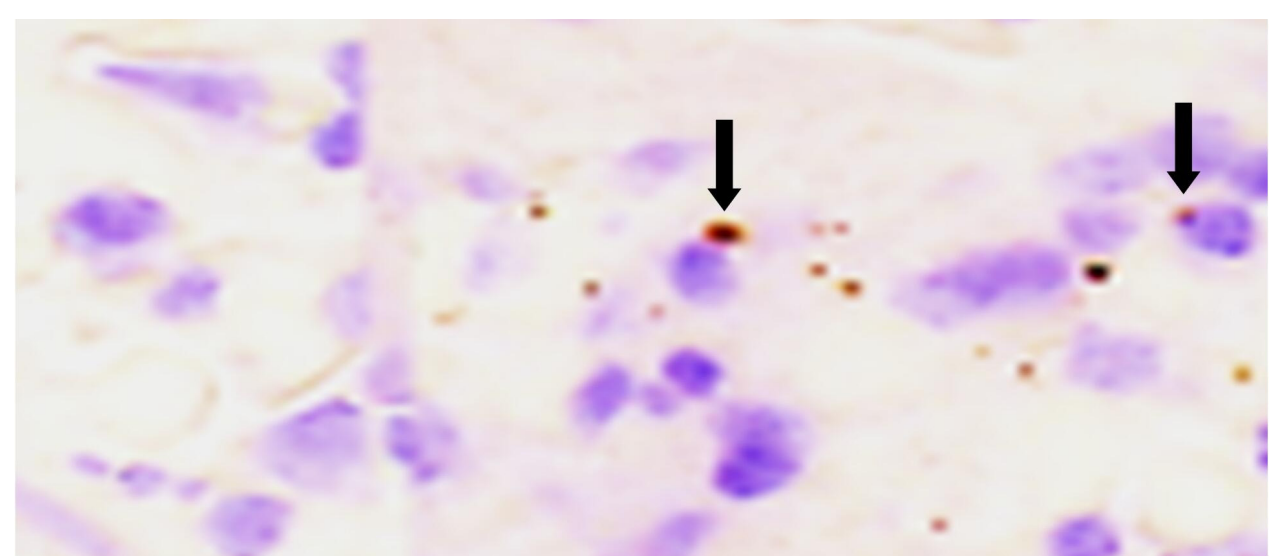

Figure 3. Chromogenic In Situ Hybridization for ALK gene signals in Emphysema (Digital 40X). Arrow shows fusion (brown) signal 
A significant high statistical difference was found between malignant and benign lesions regarding frequency of positive rearrangements signals of ALK gene $\mathrm{p}<0.001$.

The CISH test was (100\%) sensitive and (90.9\%) specific, using negative score as a cutoff point testing for ALK gene as shown in table-3.

Table 3. The distribution of ALK gene in NSCLC and Inflammatory cases

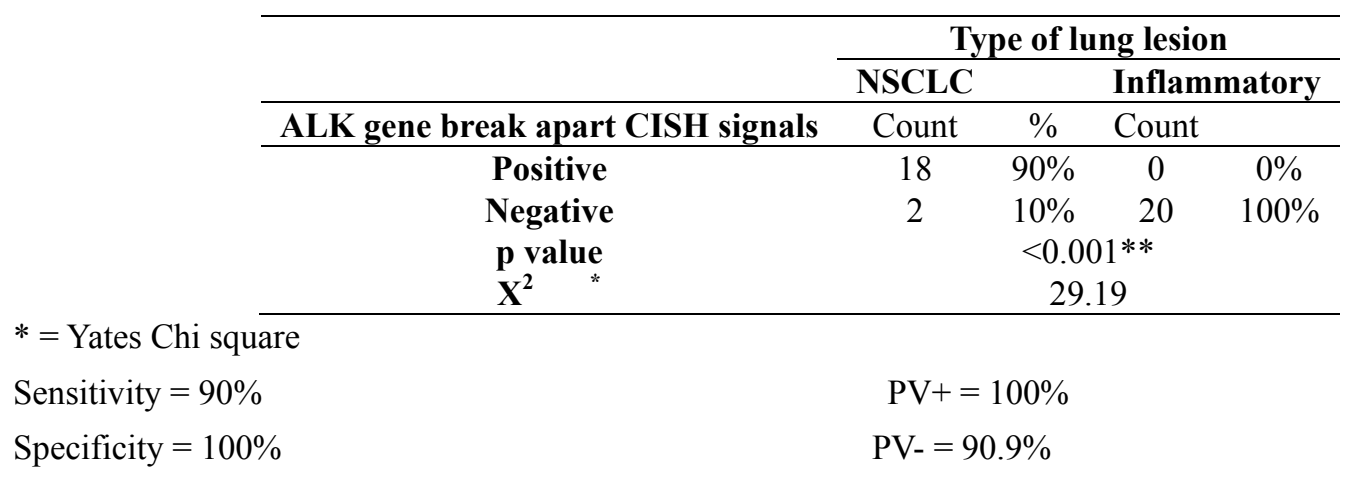

The score was divided into three levels that categorized the cases, so there were six cases in score one (1-32\%), nine cases in score two (33-67\%) and three cases in score three (68-100\%) as shown in table (1-4).

Adenocarcinoma revealed positive ALK break apart signals in $11(55 \%)$ cases and normal signal pattern in 1 (5\%) cases .Table-5. Figure-2A.

Squamous cell carcinoma revealed ALK break apart signals in 7 (35\%) cases, normal signal patterns in $1(5 \%)$ cases. Table-5. Figure-2B.

There was no significant statistical difference between adenocarcinoma and squamous cell carcinoma regarding the positive signal distribution, $\mathrm{p}>0.05$.

The difference of ALK gene signal score among different morphological types of NSCLC was statically not significant as shown in table-5. Similarly, the difference of the signal score was statically not significant among different histopathological grades of NSCLC.

According to the grade the distribution is shown in table-6, whereby pattern 1 was not seen in any of the cases of well differentiated tumor, but was shown in 1 case $(5 \%)$ of moderately differentiated tumor and $4(20 \%)$ cases of poorly differentiated tumor.

Pattern 2 was seen in $2(10 \%)$ well differentiated, $4(20 \%)$ moderately differentiated and $2(10 \%)$ poorly differentiated tumors.

Mixed pattern was seen in $2(10 \%)$ well differentiated, $2(10 \%)$ moderately differentiated and $1(5 \%)$ poorly differentiated tumors.

The difference between different grades was statistically not significant regarding the signal pattern distribution, $\mathrm{P}>0.005$.

Pearson correlation coefficient revealed a positive correlation, $\mathrm{p}<0.045$, regarding the severity of pattern of distribution with increasing grade as shown in table-6.

Table 4. Distribution of different grades of non-small cell lung cancer according to score of ALK break apart signals

\begin{tabular}{lllll}
\hline \multirow{2}{*}{ Lung cancer type } & \multicolumn{3}{l}{ ALK signal score } & \\
\cline { 2 - 5 } & Score 1 & Score 2 & Score 3 & Total \\
\hline Well Differentiated & 2 & 1 & 1 & 4 \\
$\%$ & $50.0 \%$ & $25.0 \%$ & $25.0 \%$ & $100.0 \%$ \\
Moderately Differentiated & 1 & 5 & 1 & 7 \\
$\%$ & $14.3 \%$ & $71.4 \%$ & $14.3 \%$ & $100.0 \%$ \\
Poorly Differentiated & 3 & 3 & 1 & 7 \\
$\%$ & $42.9 \%$ & $42.9 \%$ & $14.3 \%$ & $100.0 \%$ \\
P value & $0.607^{\mathrm{NS}}$ & & & \\
\hline
\end{tabular}


Table 5. Distribution of histological types of non-small cell cancer cases according to percentage score of ALK gene rearrangement

\begin{tabular}{lllll}
\hline Lung cancer type & \multicolumn{3}{l}{ ALK signal score } & \\
\cline { 2 - 5 } & Score 1 & Score 2 & Score 3 & Total \\
\hline Adenocarcinoma & 3 & 5 & 2 & 10 \\
$\%$ & $30.0 \%$ & $50.0 \%$ & $20.0 \%$ & $100.0 \%$ \\
Squamous cell Carcinoma & 3 & 4 & 1 & 8 \\
\% & $37.5 \%$ & $50.0 \%$ & $12.5 \%$ & $100.0 \%$ \\
P value & $0.894^{\mathrm{NS}}$ & & & \\
\hline
\end{tabular}

Table 6. Distribution of different grades of non-small cell lung cancer according to the pattern of ALK break apart rearrangement

\begin{tabular}{|c|c|c|c|c|c|}
\hline & No pattern & 1 mutated copy & Mixed & 2 mutated copies & Total \\
\hline & Negative & Pattern 2 & Pattern & Pattern 1 & \\
\hline Well differentiated & 1 & 2 & 2 & 0 & 5 \\
\hline Moderately differentiated & 1 & 4 & 2 & 1 & 8 \\
\hline Poorly differentiated & 0 & 2 & 1 & 4 & 7 \\
\hline Total & 2 & 8 & 5 & 5 & 20 \\
\hline Chi-square $p$ value & $0.312^{\mathrm{NS}}$ & & & & \\
\hline Pearson correlation & 0.453 & & & & \\
\hline $\mathrm{p}$ value & $0.045^{*}$ & & & & \\
\hline
\end{tabular}

\section{Discussion}

In the present study there was no significant association and correlation in NSCLC and inflammatory cases regarding sex. The reported frequencies are due to nearly equal selected sample.

This study revealed a frequency of adenocarcinoma and squamous cell carcinoma as $60 \%$ and $40 \%$ respectively, none of the cases showed large cell carcinoma. Similar study by Xie - Heshn et al (11) has shown nearly similar frequencies, $56 \%$ of adenocarcinoma, $42 \%$ of squamous cell carcinoma, and $2 \%$ of large cell carcinoma.

The difference between adenocarcinoma and squamous cell carcinoma was not significant regarding the percentage score. The score also did not differ among different grades. This is because the score percentage was calculated by counting every cell with positive break apart signal without differentiating the pattern.

The results of the histological grade of the samples in the present study were also limited by the small sample size.

Well differentiated (25\%) and moderately differentiated (40\%) were more frequently seen. This could be probably due to the fact that they can be diagnosed early before passing into a higher grade.

A study by Louise et al, showed the histological grade frequency as $35 \%$ well differentiated carcinoma, $37 \%$ moderately differentiated, and $38 \%$ poorly differentiated. These findings to some extent differ from the present study (Louise et al., 2005).

Similar studies have also implemented FISH technique for the same aims of this study, but with the addition of another fluorescent probe targeting the deleted portion of chromosome 2 in ALK rearranged NSCLC.

Sholl et al, suggested that it is important to raise a suspicion about the possibility of ALK rearrangement based on the unique clinicopathologic characteristic of NSCLC patients. They also implemented the dual testing CISH technique to identify these ALK rearrangement (Sholl et al., 2007).

The present study implemented the same technique. Results were very promising in identifying this mutation by a sensitive and highly specific test.

On application of signal pattern, discrimination between early and late process was evident (Boland et al., 2009).

The difference between grades was not significant on the application of Chi square for frequency, however the application of Pearson correlation coefficient test revealed a significant somewhat steady increase in the pattern with increasing grade. 
Schildhaus U. et al, have not studied the pattern, however, they have studied the score revealing the fact that higher scores are found in higher grades (Hans-Ulrich et al., 2013). Kwak et al, have also linked high grade NSCLC to ALK rearrangement (Kwak et al., 2010).

Definitely, studying larger sample of NSCLC could have affected the results. Hans-Schildhaus U. et al., suggested that ALK rearranged NSCLC comprise a subgroup of adenocarcinoma and Squamous cell carcinoma with distinct clinicopathologic characteristics. This group was mainly composed of young nonsmokers NSCLC patients (Hans-Ulrich et al., 2013).

This does not agree with the present study which demonstrated a higher frequency of ALK rearrangement in the NSCLC patients older than 50 years of age.

Two similar studies employing FISH technique in the demonstration of ALK rearrangement in NSCLC have found a frequency of ALK rearrangement as $90.9 \%$ and $78.6 \%$ respectively (Hans-Ulrich et al., 2013).

The present study has demonstrated the efficiency of detecting ALK gene rearrangement not only as a positive cell, but also to differentiate grades. It seems that the break apart signal is associated with the development or initiation of the carcinogenesis process. While the detection of two break apart signal was associated with a higher cancer grade (Louise et al., 2005).

The best explanation for the high detection frequency of mutated ALK found in the present work is that the detection has involved screening by a digital pathology aid. This allows for a resolution of 40X times than an ordinary microscope in which there is usually an overlapping signaling pattern that may result in false negative results.

The results of this study support the fact that patients with ALK positive rearranged NSCLC, early and advanced tumors, are better to be screened for the pattern of signals before being treated with specific targeted therapy. The presence of $20 \%$ moderately differentiated cancer with one rearranged signal might indicate that the acquisition of this mutation had occurred at later time from initial carcinogenesis.

\section{Conclusion}

Results were very promising in identifying this mutation by a sensitive and highly specific test. The ALK gene rearrangement could be an early mutation and it is needed as an initiating step for the carcinogenesis process. The presence of a double gene mutation, however, could be the cause of a higher grade cancer.

Best to the present knowledge, this is the first study of its type to be conducted in Iraq, studying ALK detection in NSCLC by CISH technique.

Detection of ALK rearrangement in the early diagnosis of NSCLC is highly sensitive and can save a lot of efforts in planning chemotherapy regimens. Therefore; it is worthy to demonstrate the presence of such rearrangement in a sample of patients with NSCLC in Iraq.

\section{References}

Boland, J., Easing, S., Vasmatzis, G. et al. (2009). Anaplastic lymphoma kinase immunoreactivity correlates with ALK gene rearrangement and transcriptional up-regulation in non-small cell lung carcinomas. Hum Pathol., 40, 1152-1158. https://doi.org/10.1016/j.humpath.2009.01.012

Christine, M., Lovly, D., Leora, H., \& William, P. (2016). Molecular Profiling of Lung Cancer. The onclogists.

Hans-Ulrich, S., Karl-Friedrich, D., Katja, S. et al, (2013). Definition of Fluorescence in Situ Hybridization score identities high and low level FGFR1 amplification types in squamous cell lung cancer, mod pathol., p95.

Kim, H., \& Choe, J. (2011). Detection of ALK gene rearrangement in non-small cell lung cancer: a comparison of fluorescence in situ hybridization and chromogenic in situ hybridization with correlation of ALK protein expression. J Thorac Oncol., 6, 1359-1366. https://doi.org/10.1097/JTO.0b013e31821cfc73

Kwak, E., B., Camidge, D., et al. (2010). Anaplastic lymphoma kinase inhibition in non-small-cell lung cancer. $N$ Engl J Med., 363, 1693-1703. https://doi.org/10.1056/NEJMoa1006448

Louise, B., Douglas, B., Donald, G., et al. (2005). Prognostic implications of molecular and immunohistochemical profiles in Primary Non small cell lung carcinoma. Clinical cancer research, 11, 232.

Morris, S., Kirstein, M., Valentine, M., Dittmer, K., Shapiro, D., \& Saltman, D., (2016). Fusion of Anaplastic lymphoma Kinase.

Sholl, L., Joerge, I., Chou, Y., et al. (2007). Validation of chromogenic in situ hybridization for detection of Modern Pathology, CISH in the diagnosis of ALK rearrangements in non small cell lung carcinoma. Mod 
Pathol, 20, 1028-1035. https://doi.org/10.1038/modpathol.3800946

Xie, G., Harshil, A., GAO, Y., \& Cheng, S. (2006). Aberrant p16 promoter hypermethylation in bronchial mucosa as a biomarker for the early detection of lung cancer. Chinese Medical Journal, 119(17), 1472.

Zochbauer-Muller, S., \& Minna, J. (2002). Molecular pathogenesis of lung cancer. Annu. Rev. Physiol, 64, 681-708. https://doi.org/10.1146/annurev.physiol.64.081501.155828

ZytoDot 2C CISH Implementation Kit and Probe, Germany, ZytoVision GmbH, Fischkai 1. 2015.

\section{Copyrights}

Copyright for this article is retained by the author(s), with first publication rights granted to the journal.

This is an open-access article distributed under the terms and conditions of the Creative Commons Attribution license (http://creativecommons.org/licenses/by/4.0/). 